\title{
Japanese Women's Age-related Differences between Controlled Force Exertion Measured by a Computer-generated Sinusoidal Waveform and a Bar Chart Display
}

\author{
Yoshinori Nagasawa ${ }^{1, *}$, Shinichi Demura ${ }^{2}$ \\ ${ }^{1}$ Department of Health and Sports Sciences, Kyoto Pharmaceutical University, Kyoto, Japan \\ ${ }^{2}$ Graduate School of Natural Science \& Technology, Kanazawa University, Kanazawa, Japan \\ *Corresponding author: ynaga@mb.kyoto-phu.ac.jp
}

Received November 13, 2014; Revised November 22, 2014; Accepted November 26, 2014

\begin{abstract}
Because developing an accurate method of measuring controlled force exertion is important, this study examined age differences in corresponding relationships between controlled force exertions measured by sinusoidal waveform and bar chart displays. Additionally, the study clarified the judgment score of the controlled force exertion's decrease. Participants were 215 right-handed female adults, aged 20-84, in three age groups: young ( $\mathrm{n}=$ 64 , mean age $24, S D=2.8$ years), middle-aged $(n=91$, mean age $43, S D=8.0$ years), and elderly ( $n=60$, mean age $68, \mathrm{SD}=6.5$ years). They matched the submaximal grip strength in their dominant hand to changing demand values displayed on a personal computer screen as either a sinusoidal waveform or a bar chart. They performed the tests three times with 1-minute inter-trial intervals. The dependent variable was the total of percentage values of differences between the demand and grip exertion values for 25 seconds. In both displays, the coefficient of variance had almost the same range in all age groups (CVSW $=25.9-32.0$, CVBC $=21.2-38.8$ ), but the elderly group showed a somewhat higher value with the bar chart. Three groups had significant correlations between scores with the sinusoidal waveform and bar chart displays $(r=0.33-0.64)$, but their values did not differ significantly among age groups. Only $0 \%-3 \%$ of the middle-aged group had scores over $1500 \% ; 23 \%-33 \%$ of the elderly group did. Furthermore, only $15 \%$ of the elderly group had scores over $1500 \%$ in both displays. There is a moderate relationship between the controlled force exerted in response to the sinusoidal waveform and bar chart displays, and it does not show age differences. In controlled force exertion, scores over $1500 \%$ in both displays are considered inferior to scores under $1500 \%$.
\end{abstract}

Keywords: controlled force exertion, hand strength, psychomotor performance, correlation coefficient

Cite This Article: Yoshinori Nagasawa, and Shinichi Demura, “Japanese Women’s Age-related Differences between Controlled Force Exertion Measured by a Computer-generated Sinusoidal Waveform and a Bar Chart Display.” American Journal of Sports Science and Medicine, vol. 2, no. 6 (2014): 212-217. doi: 10.12691/ajssm2-6-2.

\section{Introduction}

Accurate and efficient movements depend on the precise control of small muscle groups related to hands and fingers, but dynamic properties of large muscle groups, such as the magnitude of force output and endurance ability, are affected largely by neuromuscular function (Ofori, Samson, \& Sosnoff, 2010). Ranganathan, Siemionow, Sabgal, and Yue (2001) examined the effects of aging on hand function and reported that, compared with the young, the elderly were weaker in handgrip and maximum pinch force and were inferior also in ability to maintain a steady submaximal pinch force. Some have suggested that these force control properties are influenced by maturation (Deutsch \& Newell, 2001; Ofori et al., 2010), aging of neuromuscular pathways (Galganski, Fuglevand, \& Enoka, 1993), and constraints of forceoutput tasks such as the magnitude of muscular force (Sosnoff \& Newell, 2008).

To exert motor control function smoothly, information from the central (e.g., visuomotor processing) and peripheral (e.g., motor unit firing rate) nervous systems is integrated in the cerebrum (Doyon \& Benali, 2005), and proper control of movements in each motor system component is required. Thus, neuromuscular function contributes to the control of human motor performances.

In particular, skillful and efficient movements that demand feedback, such as manual dexterity and hand-eye coordination, are closely involved in the ability to control voluntary movements, i.e., controlled force exertion (Henatsch \& Langer, 1985). 
The controlled force exertion test evaluates the motor control function that coordinates force exertion during a task. Motor control function is interpreted as superior when muscle contraction and relaxation are performed smoothly in accordance with the movement of a target and with low variability of performance error and high accuracy (Brown \& Bennett, 2002).

Nagasawa and Demura (2002) focused on tracking action with submaximal exertion and developed a new test for controlled force exertion. The test makes rational objective estimation of grading, spacing (space perception), and timing, which are important elements of controlled force exertion (Nagasawa \& Demura, 2002). Furthermore, the test requires grip control (gross motor control) and hand-eye coordination, and is therefore useful for evaluating neuromuscular function in the elderly (Nagasawa, Demura, Yamaji, Kobayashi, \& Matsuzawa, 2000).

Studies that visually present controlled force exertion tasks typically use tracking paradigms (Galganski, et al., 1993; Nagasawa \& Demura, 2002). Within these paradigms, visual feedback of performance is presented through a displayed sinusoidal waveform or a bar chart.

The sinusoidal waveform signal is periodic and displayed as changes in the waveform from left to right visually and spatially over time; thus, participants can anticipate a demand value displayed by a computer monitor (i.e., a target) after the first period. Therefore, force can be exerted quickly to correspond with the demand value. On the other hand, the bar chart signal changes a large target only vertically, and hence, the participant can match its movement and easily adjust the force exerted. Consequently, a sinusoidal waveform display allows participants to use more visual information regarding performance error and more feed-forward (e.g., anticipatory) strategies during a continuous tracking task (Ofori et al., 2010).

According to Nagasawa, Demura, and Kitabayashi (2004), neuromuscular functions such as motor responsiveness, accuracy, and velocity are exerted as the difference of performance error depending on the type of demand value displayed, and their difference determines the response to sinusoidal waveform and bar chart displays. The relationships among age groups in the controlled force exertion test are considered to differ according to information received from the central and peripheral nervous systems, the effects of age in the control function concerned, and the type of displayed demand value. However, little research has examined the effects of age on relationships between the sinusoidal waveform and bar chart displays.

Because the ability to exert controlled force is generally evaluated on the basis of the error between a demand value and an exerted value (Nagasawa et al., 2000), a decrease in controlled force exertion might be evaluated on the basis increasing errors. Early detection of decline in cognitive function in the clinical and rehabilitation fields is important, but this method relies mainly on tests used in neuropsychological examination, such as those measuring processing time for movements and reaction time for actions. The controlled force test, which quantitatively estimates the circuit integrating sensory input (visual) and motor output, has not been widely used. Because the development of an accurate method for measuring controlled force exertion is desirable in both medical and rehabilitation fields, it is important to examine age differences in the relationships between the two types of display. This study's hypothesis, based on previous studies, was that differences in controlled force exertion between the two displays would decrease with age.

Therefore, this study aimed to examine Japanese women's age differences in the relationship between controlled force exertion variables measured by sinusoidal waveform and bar chart displays. To develop an accurate method of measuring controlled force exertion, the study also attempted to identify the score for determining the decrease of controlled force exertion.

\section{Materials and Method}

\subsection{Participants}

The following participants were recruited from among university students, office workers, and the elderly in Japan: 64 young females aged 20-29 (mean age 24, SD = 2.8 years), 91 middle-aged females aged 30-59 (mean age $43, \mathrm{SD}=8.0$ years), and 60 elderly females aged 60-84 (mean age 68, SD = 6.5 years). Table 1 presents their ages, grip strengths, and physical characteristics (height, body mass) by age group. On the basis of Demura et al.'s inventory (2009), all participants were regarded as righthanded.

Table 1. Physical characteristics of participants

\begin{tabular}{|c|c|c|c|c|c|c|c|c|}
\hline \multirow[t]{2}{*}{ Age group } & \multicolumn{2}{|c|}{ Age (yr) } & \multicolumn{2}{|c|}{ Height $(\mathrm{cm})$} & \multicolumn{2}{|c|}{ Body mass (kg) } & \multicolumn{2}{|c|}{ Grip strength (N) } \\
\hline & $M$ & $S D$ & $M$ & $S D$ & $M$ & $S D$ & $M$ & $S D$ \\
\hline Young (n=64) & 24.2 & 2.75 & 159.7 & 5.15 & 52.1 & 5.86 & 307.7 & 45.86 \\
\hline Middle-aged $(n=91)$ & 42.8 & 8.03 & 156.9 & 5.13 & 52.6 & 6.77 & 288.1 & 38.81 \\
\hline Elderly (n=60) & 68.2 & 6.45 & 150.6 & 6.07 & 53.7 & 8.27 & 227.4 & 61.84 \\
\hline Total $(n=215)$ & 44.3 & 17.97 & 155.9 & 6.46 & 52.7 & 6.97 & 277.3 & 57.62 \\
\hline
\end{tabular}

Note: Age ranges of young, middle-aged, and elderly groups were 20-29, 30-59, and 60-84, respectively.

For each age level, the mean values of height and body mass were similar to Japanese normative values (Laboratory Physical Education in Tokyo Metropolitan University, 1989). No participant reported previous wrist injuries or upper limb nerve damage, and all were in good health. Prior to measurement, this study's purpose and procedures were explained in detail, and written informed consent was obtained from all participants. The Ethics Committee on Human Experimentation of the Faculty of

Education, Kanazawa University, approved this experimental protocol. No participant had previously experienced a controlled force exertion test. In this study, participants over 60 were defined as elderly.

\subsection{Apparatus}

Participants wore glasses when required and stood 70 $\mathrm{cm}$ from the display. The size of the grip was set such that the participant felt comfortable squeezing it. Grip strength 
and controlled force exertion were measured with a Smedley's type handgrip mechanical dynamometer (GRIP-D5101; Takei, Tokyo, Japan), with an accuracy of $\pm 2 \%$ in the $0-979.7 \mathrm{~N}$ range (output range of $1-3 \mathrm{~V}$ ). This information was transmitted to a computer at a sampling rate of $10 \mathrm{~Hz}$ through an RS-232C data output cable (Elecom, Tokyo, Japan) after A/D conversion with a quantization bit rate of 12 bits (input range of $1-5 \mathrm{~V}$ ). Apparatus details have been previously described (Nagasawa \& Demura, 2002).

\subsection{Estimation of Maximal Grip Strength}

At the beginning of the experimental session, each participant's maximal grip strength with the dominant hand was determined. The participant was then instructed to produce her greatest possible isometric force by exerting a power grip, with the wrist in a neutral position between flexion and extension. Two five-second maximal contractions were recorded, with a minute's rest after each test. No verbal encouragement was given to participants. The greater value from the two trials was taken as the value of maximal grip strength (Nagasawa et al., 2000; Nagasawa \& Demura, 2002).

\subsection{Submaximal Controlled Force Exertion Task}

The test of controlled force exertion resembled a commonly used test of grip strength (Skelton, Greig, Davies, \& Young, 1994; Walamies \& Turjanmaa, 1993), with the exception of the exertion of a prolonged submaximal grip. Participants stood upright with the wrist in a neutral position between flexion and extension and with the elbow straight and close to the body.

As outlined in a preliminary investigation (Nagasawa \& Demura, 2002), a sinusoidal waveform and a bar chart were used for all participants. The displays simultaneously showed both demand value and actual grip strength; however, these variables' method of display differed. That is, both demand value and changes in the actual gripexertion value were displayed as changes in the sinusoidal waveform from left to right visually and spatially with time, and as vertical changes in the bar for the bar chart.

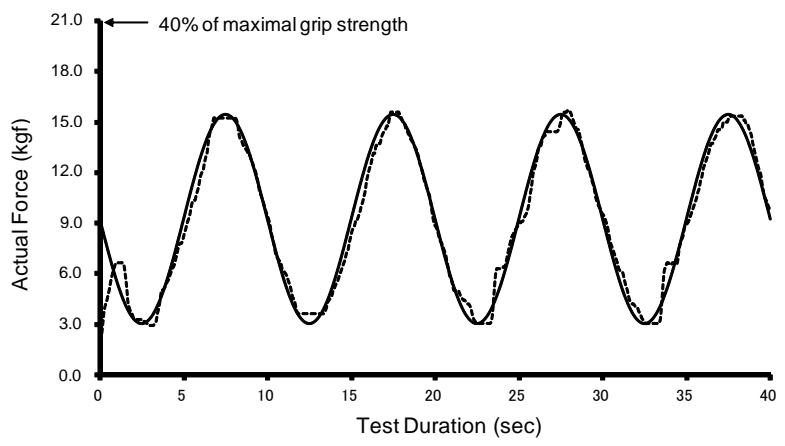

Figure 1. Sinusoidal waveform display $(100 \mathrm{~mm} \times 140 \mathrm{~mm})$ of the demand value. The solid waveform (A) shows the demand value and the dashed waveform (B) is the exertion value of grip strength. The test was to fit line B (exertion value of grip strength) to line A (demand value), which varied in the range of $5 \%-25 \%$ of maximal grip strength. The length on the display is $33 \mathrm{~mm}$, top to bottom. Frequency of change in demand value is $0.1 \mathrm{~Hz}$. The test time was 40 seconds for each trial. After the initial 15 seconds of the 40-second trial, the coordinated exertion of force was calculated using the data obtained from the following 25 seconds
The demand values of the sinusoidal waveform and bar chart varied over 40 seconds at a frequency of $0.1 \mathrm{~Hz}$ and $0.3 \mathrm{~Hz}$, respectively (Nagasawa \& Demura, 2009; Nagasawa, Demura, \& Nakata, 2003). The participants attempted to minimize the difference between the demand value and the value of their grip strength as presented on the computer display. Figure 1 and Figure 2 show the sinusoidal waveform and bar chart displays, respectively. Participants in a preliminary experiment were able to track the demand values in both displays.

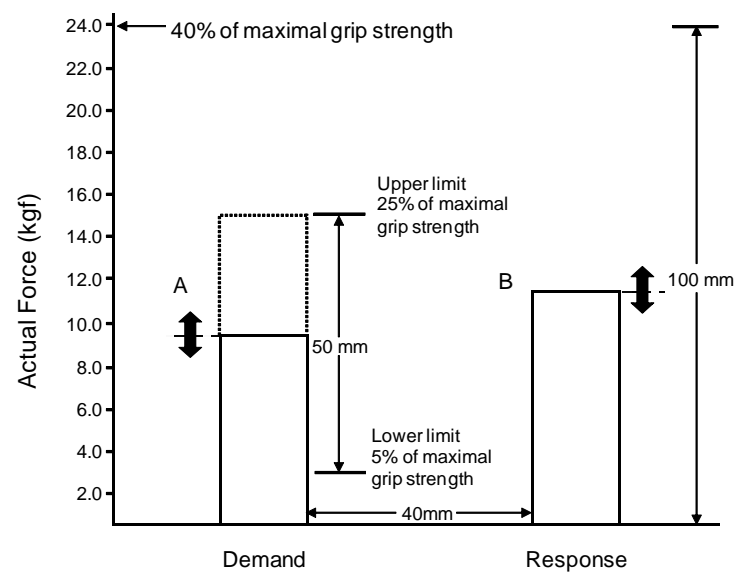

Figure 2. Bar chart display $(100 \mathrm{~mm} \times 140 \mathrm{~mm})$ of the demand value. Bar chart display (100 mm $\times 140 \mathrm{~mm}$ ) of the demand value. Left bar (A) shows the demand value and right bar (B) is the exertion value of grip strength. The test was to fit line B (exertion value of grip strength) to line A (demand value), which varied in a span of $50 \mathrm{~mm}$ on the display. The test time was $40 \mathrm{~s}$ for each trial. After the initial 15 seconds of the 40second trial, the controlled force exertion was calculated using the data from the following 25 seconds.. Actual force was shown on the right of the display

Relative, not absolute, demand values were used because individuals differ in physical fitness and muscular strength. The relative demand value varied by approximately $5 \%-25 \%$ of maximal grip strength (Nagasawa et al., 2003, 2004). All participants were presented with the same shape of demand function. The software program was designed to present relative demand values within a constant range on the computer display. The demand value for the sinusoidal waveform and the bar chart targets varied cyclically (see Figure 1 and Figure 2).

The visual displays were randomly presented to each participant within each display type block. Within each unique force display condition, the participant performed three trials after one practice trial. Demand values in the displays were tracked, and performance was measured by the sum of the percentage values of differences between the demand value and grip exertion value. To minimize the effect of fatigue, a 1-minute rest period was provided after each trial, and a 3-minute rest period was provided after each display condition. Six trials were performed: three sinusoidal waveform and bar chart trials at each frequency and one relative demand level.

The sum of the percentage value of differences between the demand value and grip strength was used to estimate controlled force exertion scores (Nagasawa \& Demura, 2002), with smaller differences indicating a better ability to control force exertion. The duration of each trial was 40 seconds, and the controlled force exertion scores in each display condition were estimated from data of three trials, 
excluding the first 15 seconds of each trial, as in the previous study of Nagasawa et al. (2000). The mean of the second and third trials was used for the analysis (Nagasawa, et al., 2004).

\subsection{Statistical Analysis}

Data were analyzed with SPSS Version 17.0 for Windows (SPSS Inc., Tokyo, Japan). The data were reported using ordinary statistical methods, including mean (M) and standard deviation ( \pm standard deviation, SD).

A one-way analysis of variance on age, grip strength, and physical characteristics (height, body mass) was conducted to examine significant differences among age groups. When a significant effect was found, a multiplecomparison test was conducted using Tukey's honestly significant difference (HSD) method for pair-wise comparisons.

For each age group, correlation analyses were employed to identify associations between the controlled force exerted in response to sinusoidal waveform and bar chart displays. In addition, coefficients of variance were calculated to examine individual differences between age groups. An alpha level of 0.05 was considered significant for all tests.

\section{Results}

The means of age revealed significant differences among age groups. The young and middle-aged groups had significantly greater means of standing height and grip strength than the elderly group. Table 2 shows the means of each age group for the sinusoidal waveform and the bar chart, and correlations between the two displays. The coefficient of variance had nearly the same range in all age groups for both displays (CVSW $=25.9-32.0$, CVBC
= 21.2-38.8), but the elderly group showed a somewhat high value (38.8) for the bar chart.

Figure 3 shows scatter plots for the controlled force exertion scores of the two displays. Significant correlations were observed between the sinusoidal waveform and bar chart in the young, middle-aged, and elderly groups. These correlations did not differ significantly among age groups.

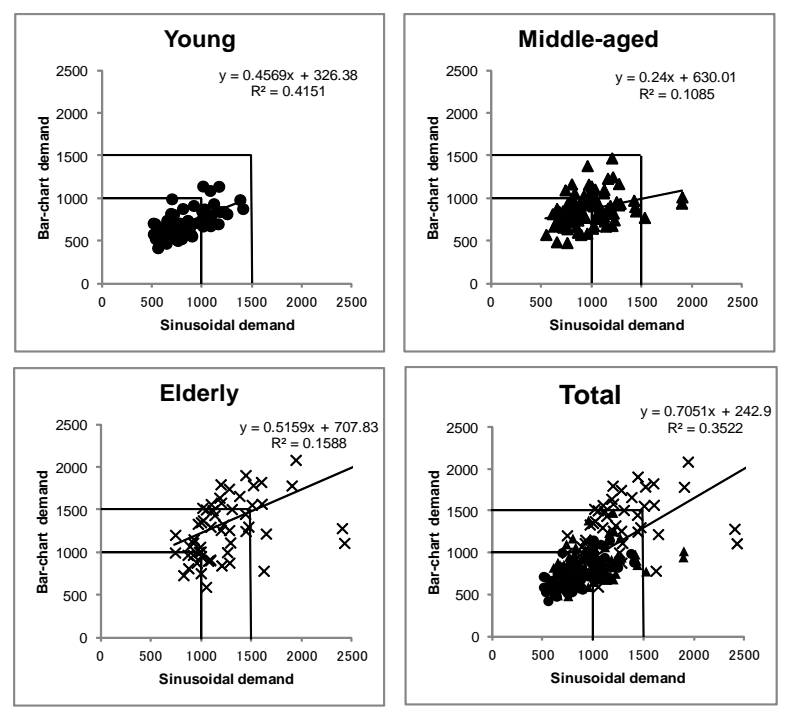

Figure 3. Scatter plots by age group for the controlled force exertion score in the sinusoidal and bar chart demands

Table 3 shows the frequency and ratio of the controlled force exertion scores for the two displays. Scores over $1500 \%$ (Nagasawa \& Demura, 2007) in the sinusoidal and bar chart displays were found in only a few individuals in the middle-aged group, but in, respectively, 23\% and 33\% of participants in the elderly group. Participants with scores over $1500 \%$ for both displays were found only in the elderly group (15\%).

Table 2. Means, standard deviations, coefficient of variance and correlations by age group for the controlled force exertion test in the sinusoidal and bar-chart demands

\begin{tabular}{|c|c|c|c|c|c|c|c|c|}
\hline \multirow[t]{2}{*}{ Age group } & \multicolumn{3}{|c|}{ Sinusoidal demand (\%) } & \multicolumn{3}{|c|}{ Bar-chart demand (\%) } & \multirow[b]{2}{*}{$r$} & \\
\hline & $M$ & $S D$ & $C V$ & $M$ & $S D$ & $C V$ & & \\
\hline Young (n=64) & 812.02 & 227.53 & 28.02 & 697.42 & 161.36 & 23.14 & 0.64 & * \\
\hline Middle-aged $(n=91)$ & 969.89 & 251.45 & 25.93 & 862.76 & 183.16 & 21.23 & 0.33 & * \\
\hline Elderly (n=60) & 1279.14 & 409.60 & 32.02 & 1367.71 & 530.21 & 38.77 & 0.40 & * \\
\hline Total $(n=215)$ & 1009.21 & 347.57 & 34.44 & 954.46 & 412.91 & 43.26 & 0.59 & * \\
\hline
\end{tabular}

Note: Age ranges of young, middle-aged, and elderly groups were 20-29, 30-59, and 60-84, respectively. * $<<0.05$

Table 3. Frequency and ratio by age group for the controlled force exertion test in the sinusoidal and bar-chart demands

\begin{tabular}{|c|c|c|c|c|c|c|c|c|c|}
\hline \multirow[t]{2}{*}{ Age group } & \multicolumn{3}{|c|}{ Sinusoidal demand score } & \multicolumn{3}{|c|}{ Bar-chart demand score } & \multicolumn{3}{|c|}{$\begin{array}{l}\text { Both Sinusoidal and Bar-chart demand } \\
\text { score }\end{array}$} \\
\hline & $\begin{array}{c}\text { under } \\
1000\end{array}$ & $\begin{array}{c}1000- \\
1500\end{array}$ & $\begin{array}{l}\text { over } \\
1500\end{array}$ & $\begin{array}{c}\text { under } \\
1000\end{array}$ & $\begin{array}{c}1000- \\
1500\end{array}$ & $\begin{array}{l}\text { over } \\
1500\end{array}$ & under 1000 & $1000-1500$ & over 1500 \\
\hline \multirow[t]{2}{*}{ Young $(n=64)$} & 48 & 16 & 0 & 61 & 3 & 0 & 61 & 3 & 0 \\
\hline & (75.0) & $(25.0)$ & $(0.0)$ & (95.3) & $(4.7)$ & $(0.0)$ & (95.3) & $(4.7)$ & $(0.0)$ \\
\hline \multirow[t]{2}{*}{ Middle-aged (n=91) } & 55 & 33 & 3 & 74 & 17 & 0 & 83 & 8 & 0 \\
\hline & $(60.4)$ & (36.3) & $(3.3)$ & (81.3) & $(18.7)$ & $(0.0)$ & $(91.2)$ & $(8.8)$ & $(0.0)$ \\
\hline \multirow[t]{2}{*}{ Elderly (n=60) } & 17 & 29 & 14 & 15 & 25 & 20 & 39 & 12 & 9 \\
\hline & (28.3) & (48.3) & (23.3) & (25.0) & $(41.7)$ & (33.3) & $(65.0)$ & (20.0) & $(15.0)$ \\
\hline
\end{tabular}

23.14). Overall, however, participants in this study formed

\section{Discussion}

The coefficient of variance for controlled force exertion with the bar chart was somewhat higher in the elderly group than in the other two groups (38.77 vs. 21.23 and a homogeneous group for controlled force exertion. Bemben, Massey, Bemben, Misner, and Boileau (1996) reported that the elderly show a noticeable decline in peripheral muscle activity when compared with young people on the basis of the measurement of muscular endurance using intermittent grip strength. 
Voelcker-Rehage and Alberts (2005) reported that young participants are superior to elderly participants in the changing force tracking task. Compared to the young, the elderly are considered to have inferior controlled force exertion (i.e., peripheral muscular responses to the changing target and the exertion of neuromuscular function). Because the sinusoidal waveform signals are periodic and displayed as changes in waveform from left to right visually and spatially with time, the sinusoidal waveform display is easy to anticipate after the first period. It requires participants to use more visual information concerning performance errors and more feed-forward (e.g., anticipatory) strategies than the bar chart display (Ofori et al., 2010). Also, with the sinusoidal demand, individuals can continually change and regulate their force exertion. In contrast, with the bar chart demand, they exert a constant force level at a higher frequency than with the sinusoidal waveform display. This might explain why greater individual differences in the elderly group than in the other age groups were found with the bar chart display.

On the basis of these differences between the two displays, it was hypothesized that the relationship between controlled force exertion values would decrease with age. However, contrary to the hypothesis, significant correlations were observed between the sinusoidal waveform and bar chart displays in all age groups, and no significant differences in correlations were found between age groups. From these results, it is inferred that at all ages, participants can correctly regulate their controlled grip force exertion in a pursuit task, regardless of the displays' difference.

The functional role in movement performance might differ according to the region of the nervous system controlling each movement. The cerebellum is generally associated with skilled motor behaviors, and the basal ganglia, particularly the striato-nigral system, is associated with actual motor behavior (Doyon \& Benali, 2005).

Reports by several researchers (Nagasawa \& Demura, 2010, 2011; Ofori et al., 2010) revealed that aged-related differences are greater with pursuit movements and that controlled force exertion decreases with age. The present test was performed using submaximal muscular exertion with a moderate cycle $(0.1 \mathrm{~Hz}$ and $0.3 \mathrm{~Hz})$ of changing demand values. Success in this test strongly requires handeye coordination, and exertion of this function is controlled by feedback, e.g., sensing force exertion and target matching. The magnitude and dynamic properties of force output indicate neuromuscular function (Ofori et al., 2010). Muscular strength decreases with changes of neuromuscular pathways and muscle fiber composition, spinal motor neuron apoptosis (Galganski et al., 1993); muscle atrophies with age (Cauley et al., 1987).

Although no participants scored over $1500 \%$ in the young group for either display, zero (0\%) and three (3\%) individuals in the middle-aged group, and fourteen (23\%) and twenty (33\%) in the elderly group scored above $1500 \%$. That is, the frequency of participants scoring over $1500 \%$ increased with age. Some of the elderly (15\%) had scores over $1500 \%$ in both displays (see Figure 3 and Table 3).

Nagasawa and Demura (2011) reported that, for controlled force exertion, the rate of decrease is remarkable after the age of 50, and scores over $1500 \%$ are very inferior in the 5-point scale according to age level. To summarize, since no participants scoring over $1500 \%$ in both displays were found in the middle-aged group and only a few in the elderly group, those scoring over $1500 \%$ in both displays were considered significantly inferior in controlled force exertion. Hence, appropriate measures must be taken to improve the controlled force exertion of people with greater scores than $1500 \%$ in both displays, and to intervene early if this problem is detected in middle-aged people.

Therefore, it is necessary to pay attention to a score over a fixed value (1500\%) or an abnormally high score in either of the displays because this suggests a marked decrease in controlled force exertion. Thus, individuals with poor controlled force exertion might also be identified according to the relationship between scores achieved in each display.

This study's participants were healthy, active female adults aged 20-84, with mean maximal grip strength of more than $277.3 \mathrm{~N}$. A follow-up study will be necessary to clarify the relationship between performances using the two displays and to compare the controlled force exertion from both displays between healthy individuals and individuals with arm and muscular nervous dysfunction.

\section{Conclusions}

In conclusion, measurements of controlled force exertion using the sinusoidal and bar chart displays show a significant relationship between relative grip exertion values. Furthermore, these relationships do not differ as a function of age. Individuals with scores over 1500\% in both displays are considered quite inferior in controlled force exertion. Those with poor controlled force exertion might also be identified by the relationship between scores achieved in each display.

\section{Acknowledgements}

This study was supported in part by a Grant-in-Aid for Scientific Research (project number 20500506) to Y. Nagasawa from the Ministry of Education, Science and Culture of Japan.

\section{Statement of Competing Interests}

The authors have no competing interests.

\section{References}

[1] Bemben, M.G., Massey, B.H., Bemben, D.A., Misner, J.E. and Boileau, R.A. "Isometric intermittent endurance of four muscle groups in men aged 20-74 yr," Medicine and Science in Sports and Exercise, 28, 145-154, 1996.

[2] Brown, S.W. and Bennett, E.D. "The role of practice and automaticity in temporal and nontemporal dual-task performance,” Psychological Research, 66, 80-89, 2002.

[3] Cauley, J.A., Petrini, A.M., LaPorte, R.E., Sandler, R.B., Bayles, C.M., Robertson, R.J. and Slemenda, C.W. "The decline of grip strength in the menopause: relationship to physical activity, estrogen use and anthropometric factors," Journal of Chronic Diseases, 40, 115-120, 1987. 
[4] Demura, S., Sato, S. and Nagasawa, Y. "Re-examination of useful items for determining hand dominance," Gazzeta Medica Italiana-Archives of Science Medicine, 168, 169-177, 2009.

[5] Deutsch, K.M., and Newell, K.M. "Age differences in noise and variability of isometric force production,” Journal of Experimental Child Psychology, 80, 392-408, 2001.

[6] Doyon, J. and Benali, H. "Reorganization and plasticity in the adult brain during learning of motor skills," Current Opinion Neurobiology, 15, 161-167, 2005.

[7] Galganski, M.E., Fuglevand, A.J. and Enoka, R.M. "Reduced control of motor output in a human hand muscle of elderly subjects during submaximal contractions," Journal of Neurophysiology, 69, 2108-2115, 1993.

[8] Henatsch, H. D. and Langer, H.H. "Basic neurophysiology of motor skills in sport: a review,” International Journal of Sports Medicine, 6, 2-14, 1985.

[9] Laboratory of Physical Education, Tokyo Metropolitan University. (Ed.) Physical Fitness Standards of Japanese People (4th ed.) Fumaido, Tokyo, 1986. [in Japanese]

[10] Nagasawa, Y. and Demura, S. "Development of an apparatus to estimate coordinated exertion of force," Perceptual and Motor Skills, 94, 899-913, 2002.

[11] Nagasawa, Y. and Demura, S. "Age and sex differences in controlled force exertion measured by a computing bar chart target-pursuit system," Measurement in Physical Education and Exercise Science, 13, 140-150, 2009.

[12] Nagasawa, Y. and Demura, S. "Provisional norms by age group for Japanese women on the controlled force exertion test using a quasi-random display,” Perceptual and Motor Skills, 110, 613-624, 2010.

[13] Nagasawa, Y. and Demura, S. "Provisional standard by age groups of the controlled force exertion test using a sinusoidal display in Japanese females,” The_Kyoyakuronsyu, 18, 37-46, 2011.
[14] Nagasawa, Y., Demura, S. and Kitabayashi, T. "Concurrent validity of tests to measure the coordinated exertion of force by computerized target-pursuit,” Perceptual and Motor Skills, 98, 551-560, 2004.

[15] Nagasawa, Y., Demura, S. and Nakata, M. "Reliability of a computerized target-pursuit system for measuring coordinated exertion of force,” Perceptual and Motor Skills, 96, 1071-1085, 2003.

[16] Nagasawa, Y., Demura, S., Yamaji, S., Kobayashi, H. and Matsuzawa, J. "Ability to coordinate exertion of force by the dominant hand: comparisons among university students and 65- to 78-year-old men and women," Perceptual and Motor Skills, 90, 995-1007, 2000.

[17] Ofori, E., Samson, J. M. and Sosnoff, J. J. "Age-related differences in force variability and visual display,” Experimental Brain Research, 203, 299-306, 2010.

[18] Ranganathan, V. K., Siemionow, V., Sahgal, V. and Yue, G. H. "Effects of aging on hand function," Journal of the American Geriatric Society, 49, 1478-1484, 2001.

[19] Skelton, D. A., Greig, C. A., Davies, J. M. and Young, A. "Strength, power and related functional ability of healthy people aged 65-89 years,” Age and Ageing, 23, 371-377, 1994.

[20] Sosnoff, J. J. and Newell, K. M. “Age-related loss of adaptability to fast time scales in motor variability,” Journal of Gerontology B: Psychological Science and Social Science, 63, 344-352, 2008.

[21] Voelcker-Rehage C., Alberts J.L. "Age-related changes in grasping force modulation,” Experimental Brain Research, 166, 61-70, 2005.

[22] Walamies, M. and Turjanmaa, V. "Assessment of the reproducibility of strength and endurance handgrip parameters using a digital analyzer,” European_Journal of Applied Physiology and Occupational Physiology, 67, 83-86, 1993. 